\title{
ROTHE APPROACH TO THE EXISTENCE OF SOLUTION OF AN ABLATION-TRANSPIRATION COOLING CONTROL SYSTEM ${ }^{1}$
}

\author{
GUO BAO $\mathrm{ZHU}^{2}$ \\ Institute of Systems Science \\ Academia Sinica \\ Beijing, CHINA
}

\begin{abstract}
A one dimensional ablation model with transpiration cooling control and nonlinear effect is studied, which is a distributed parameter control system with moving boundary and both governing equation and boundary conditions involving control variable. A solution is given by a Rothe approximation scheme.

Key words: Ablation-transpiration cooling, distributed parameter control system, moving boundary, Rothe approximation.
\end{abstract}

AMS (MOS) subject classifications: $\quad$ 49J05, 49L01, 49M99.

\section{INTRODUCTION}

When a space vehicle flies quickly through the air, the high temperature due to the friction between the front surface of the vehicle and air may cause ablation of the material which will cause damage to the structure of the vehicle. The ablation is even serious for the sideways of launching of the electromagnetic gun. In engineering, a thermal shield must be designed to prevent this event [1-4]. In [1,2], it is realized by coupling a transpiration cooling control design, which can be demonstrated by a one dimensional version of a solid thermal shield as shown in Figure 1.

\footnotetext{
${ }^{1}$ Received: October, 1992. Revised: March, 1993.

${ }^{2}$ This work was carried out with the aid of a grant from National Postdoctoral Foundation of China.
} 


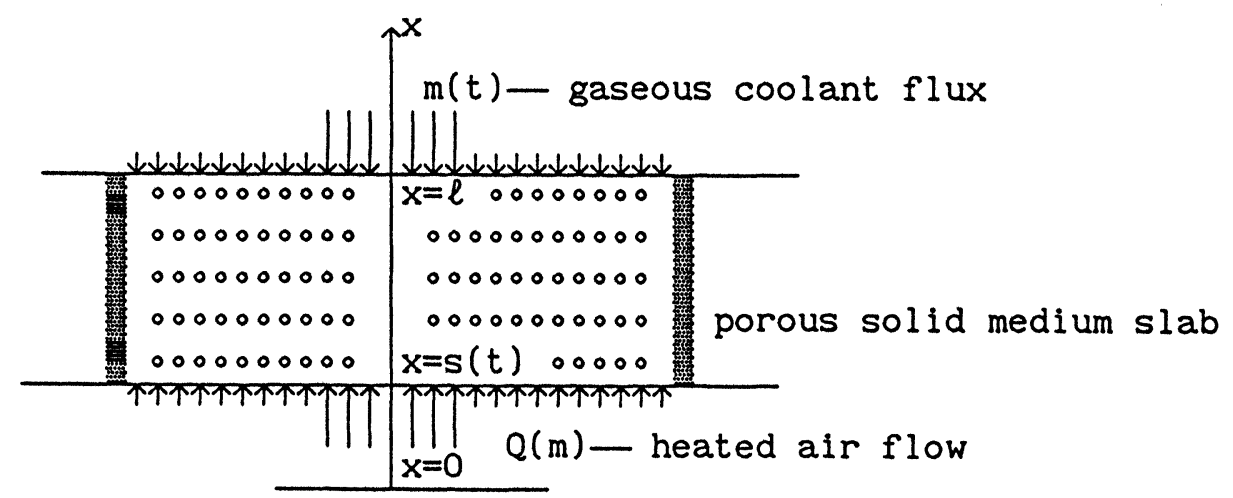

Figure 1: Schematic representation of the transpiration cooling control system of a porous medium slab

The thermal shield consists of a porous solid structure of thickness $\ell$. The gaseous coolant is inputted at $x=\ell$ and the heated air flow enters at $x=0$. The coolant flows through the air hole of the slab and enters into the heater air flow $Q(m)$, a specified flow function of the coolant flux $m(t)$ (mass of coolant per unit time flowing through per unit area) on the outer layer of the structure $[1,2]$ :

$$
Q(m)=\bar{q} \Psi, \bar{q}=q_{0}\left(1-\frac{h_{w}}{h_{r}}\right)
$$

where $q_{0}$ is the theoretical heat flow, $h_{r}$ is the recovery enthalpy, $h_{w}=c_{\rho} u_{w}$ is the enthalpy of outer surface. The blocking coefficient $\Psi$ is different for different coolants:

$$
\begin{aligned}
& \text { for helium, } \Psi=1-0.724 \frac{h_{e}}{q_{0}} m-0.13\left(\frac{h_{e}}{q_{0}} m\right)^{2} \\
& \text { for water, } \Psi=1-1.04 m \Delta h_{i} / q_{0} .
\end{aligned}
$$

When the temperature of the front face exceeds the melting temperature $u_{m}$ of the material, the outer layer melts and recedes to the new position $x=s(t)$ after time $t$ (Figure 1). Denoting by $u(x, t)$ the temperature of the medium at the space-time point $(x, t)$ and denoting by $u_{c}$ the temperature of the coolant inside the tank, Yang [1] formulated this process as the following partial differential equation with a moving boundary:

$$
\left\{\begin{array}{c}
\frac{\partial u(x, t)}{\partial t}=\alpha^{2} \frac{\partial u^{2}(x, t)}{\partial x^{2}}+\beta(t) \frac{\partial u(x, t)}{\partial x}+s^{\prime}(t) \frac{\ell-x}{\ell-s(t)} \frac{\partial u(x, t)}{\partial x}, s(t)<x<\ell \\
-\kappa \frac{\partial u(\ell, t)}{\partial x}=m(t)\left(\Delta h_{N}-\Delta h_{c}\right)=m(t) c_{p L}\left(u(\ell, t)-u_{c}\right) \\
\rho L s^{\prime}(t)-\kappa \frac{\partial u(s(t), t)}{\partial x}=Q(m(t))
\end{array}\right.
$$


where $L$ is the latent heat of melting. Symbols $\Delta h_{N}=c_{p L} u_{N}, \Delta h_{c}=c_{p L} u_{c}$ denote the enthalpy of the coolant at inner face and at tank storing coolant, respectively. The thermal conductivity $\kappa$, density $\rho$ and the specific heat $c_{\rho}$ of the ablation material are constants, $c_{p L}$ is the specific heat of the coolant, $\alpha^{2}=\kappa /\left(\rho c_{\rho}\right)$ and $\beta(t)=c_{p L} /\left(\rho c_{\rho}\right) m(t)$.

Since the melted part is broken away (by wind for example) from material immediately after melting, the following Signorini boundary condition is necessary (see [5]):

$$
u(s(t), t) \leq u_{m}, s^{\prime}(t) \geq 0,\left[u_{m}-u(s(t), t)\right] s^{\prime}(t)=0 .
$$

Observing that $u_{w}=u(s(t), t)$ and $u_{N}=u(\ell, t)$, we finally formulate the ablation transpiration cooling control process as the following Stefan-Signorini problem:

$$
\left\{\begin{array}{c}
u_{t}(x, t)=\alpha^{2} u_{x x}(x, t)+\beta(t) u_{x}(x, t)+s^{\prime}(t) \frac{\ell-x}{\ell-s(t)} u_{x}(x, t), s(t)<x<\ell, \\
\alpha^{2} u_{x}(\ell, t)+\beta(t) u(\ell, t)=\beta(t) u_{c}, u_{c}>0 \\
u(s(t), t) \leq u_{m}, s^{\prime}(t) \geq 0,\left[u_{m}-u(s(t), t)\right] s^{\prime}(t)=0 \\
s^{\prime}(t)=\frac{\kappa}{\rho L}\left[u_{x}(s(t), t)-a_{1}(\beta(t)) u(s(t), t)+a_{2}(\beta(t))\right], s(0)=0
\end{array}\right.
$$

where

$$
a_{1}(\beta(t))=\frac{q_{0} c}{\kappa h_{r}} \Psi, a_{2}(\beta(t))=\frac{q_{0}}{\kappa} \Psi .
$$

The initial condition $u(x, 0)=u_{0}(x)$ and the function $\beta(t)$ should satisfy the consistency condition:

$$
\left\{\begin{array}{c}
u_{0} \in C^{2}[0, \ell], u_{c} \leq u_{0} \leq u_{m}, \beta(t) \in C^{1}[0, \infty), Q(\beta(t)) \in C^{1}[0, \infty), \\
u_{c} \leq u_{0}(x) \leq u_{m}, \beta(t) \geq 0, \beta(t) \leq \beta_{m}, \alpha^{2} u_{0}^{\prime}(\ell)+\beta(0) u_{0}(\ell)=u_{c} \beta(0), \\
u_{0}(0) \leq u_{m}, u_{0}^{\prime}(0)-a_{1}(\beta(0)) u_{0}(0)+a_{2}(\beta(0)) u_{0}(0) \geq 0, \\
{\left[u_{0}(0)-u_{m}\right]\left[u_{0}^{\prime}(0)-a_{1}(\beta(0)) u_{0}(0)+a_{2}(\beta(0)) u_{0}(0)\right]=0 .}
\end{array}\right.
$$

To simplify notation, let

$$
v(x, t)=u_{m}-u(x, t), v_{0}(x)=u_{m}-u_{0}(x) .
$$

Then problem (4) can be written in the form

$$
\left\{\begin{array}{c}
v_{t}(x, t)=\alpha^{2} v_{x x}(x, t)+\beta(t) v_{x}(x, t)+s^{\prime}(t) \frac{\ell-x}{\ell-s(t)} v_{x}(x, t), \\
\alpha^{2} v_{x}(\ell, t)+\beta(t) v(\ell, t)=q \beta(t), q=u_{m}-u_{c}>0, \\
v(s(t), t) \geq 0, s^{\prime}(t) \geq 0, v(s(t), t) s^{\prime}(t)=0, \\
v(x, 0)=v_{0}(x), \\
s^{\prime}(t)=\frac{\kappa}{\rho L}\left[-v_{x}(s(t), t)+a_{1}(\beta(t)) v(s(t), t)+Q(\beta(t))\right]
\end{array}\right\}
$$

where $Q(\beta(t))=\frac{q_{0}}{\kappa}\left(1-\frac{c^{\rho}}{h_{r}} u_{m}\right) \Psi>0$. 
In the paper, the symbol $\|\cdot\|$ is used for the $C^{0}$ norm of a continuous function.

\section{A FIXED PROBLEM}

For any fixed time $T>0$, and a function

$$
s(t) \in C^{1}[0, T], s(0)=0, s^{\prime}(t) \geq 0, s(T)<\ell,
$$

the standard transformation

$$
y=\frac{x-s(t)}{\ell-s(t)} \ell, \vartheta(y, t)=v(x, t)
$$

will map $[s(t), \ell]$ onto $[0, \ell]$ and the fixed problem (8) becomes a fixed domain problem:

$$
\left\{\begin{array}{c}
\vartheta_{t}(y, t)=\alpha^{2}\left(\frac{\ell}{\ell-s(t)}\right)^{2} \vartheta_{y y}(y, t)+\left[\beta(t) \frac{\ell}{\ell-s(t)}+2 s^{\prime}(t) \frac{\ell-y}{\ell-s(t)}\right] \vartheta_{y}(y, t), \\
\alpha^{2} \frac{\ell}{\ell-s(t)} \vartheta_{y}(\ell, t)+\beta(t) \vartheta(\ell, t)=q \beta(t), \\
\vartheta(0, t) \geq 0,-\frac{\ell}{\ell-s(t)} \vartheta_{y}(0, t)+a_{1}(\beta(t)) \vartheta(0, t)+Q(\beta(t)) \geq 0, \\
\vartheta(0, t)\left[-\frac{\ell}{\ell-s(t)} \vartheta_{y}(0, t)+a_{1}(\beta(t)) \vartheta(0, t)+Q(\beta(t))\right]=0 .
\end{array}\right.
$$

Let

$$
0=t_{0}<t_{1}<t_{2}<\ldots<t_{N}=T, t_{n}-t_{n-1}=\Delta t=\frac{T}{N}
$$

be a uniform partition of $[0, T]$. Considering

$$
\beta_{n}=\beta\left(t_{n}\right), s_{n}=s\left(t_{n}\right),
$$

and applying the Rothe approximation scheme to (11), we obtain a boundary value problem as follows:

$$
\left\{\begin{array}{c}
\frac{\vartheta_{n}(y) \vartheta_{n-1}(y)}{\Delta t}=\alpha^{2}\left(\frac{\ell}{\ell-s_{n}}\right)^{2} \vartheta_{n}^{\prime \prime}(y)+\left[\beta_{n} \frac{\ell}{\ell-s_{n}}+2 \frac{s_{n}-s_{n-1}}{\Delta t} \frac{\ell-y}{\ell-s_{n}}\right] \vartheta_{n}^{\prime}(y), \\
\alpha^{2} \frac{\ell}{\ell-s_{n}} \vartheta_{n}^{\prime}(\ell)+\beta_{n} \vartheta_{n}(\ell)=q \beta_{n}, \vartheta_{0}(y)=v_{0}(y), \\
\vartheta_{n}(0) \geq 0,-\frac{\ell}{\ell-s_{n}} \vartheta_{n}^{\prime}(0)+a_{1}\left(\beta_{n}\right) \vartheta_{n}(0)+Q\left(\beta_{n}\right) \geq 0 \\
\vartheta_{n}(0)\left[-\frac{\ell}{\ell-s_{n}} \vartheta_{n}^{\prime}(0)+a_{1}\left(\beta_{n}\right) \vartheta_{n}(0)+Q\left(\beta_{n}\right)\right]=0 .
\end{array}\right.
$$

Using the transformation

$$
x=s_{n}+\frac{\ell-s_{n}}{\ell} y, \quad v_{n}(x)=\vartheta_{n}(y)
$$

we finally obtain the Rothe approximation scheme of the fixed problem (8): 


$$
\left\{\begin{array}{c}
\frac{v_{n}(x)-v_{n-1}(\vartheta(x))}{\Delta t}=\alpha^{2} v_{n}^{\prime \prime}(x)+\left[\beta_{n}+2 \frac{s_{n}-s_{n-1}}{\Delta t} \frac{\ell-x}{\ell-s_{n}} v_{n}^{\prime}(x), s_{n}<x<\ell,\right. \\
\alpha^{2} v_{n}^{\prime}(\ell)+\beta_{n} v_{n}(\ell)=q \beta_{n} \\
v_{n}\left(s_{n}\right) \geq 0,-v_{n}^{\prime}\left(s_{n}\right)+a_{1}\left(\beta_{n}\right) v_{n}\left(s_{n}\right)+Q\left(\beta_{n}\right) \geq 0 \\
v_{n}\left(s_{n}\right)\left[-v_{n}^{\prime}\left(s_{n}\right)+a_{1}\left(\beta_{n}\right) v_{n}\left(s_{n}\right)+Q\left(\beta_{n}\right)\right]=0 \\
v_{0}(x)=v_{m}-v_{0}(x), \vartheta(x)=\frac{\ell-s_{n-1}}{\ell-s_{n}} x-\frac{s_{n}-s_{n-1}}{\ell-s_{n}} \ell
\end{array}\right.
$$

Lemma 1: There exists a unique solution $\left\{v_{n}(x), 1 \leq n \leq N\right\}$ to (14) and

$$
0 \leq v_{n}(x) \leq q, c_{1} \leq v_{n}^{\prime}(x) \leq c_{2}
$$

where

$$
c_{1}=\min v_{0}^{\prime}(x), \quad c_{2}=\max \left\{\left\|v_{0}\right\|, q \beta_{M} / \alpha^{2}, q\left\|a_{1}\right\|+\|Q\|\right\}
$$

Proof: Equation (14) can be solved by the method of invariant imbedding (sweep method) (see [9]):

$$
v_{n}^{\prime}(x)=R_{n}(x) v_{n}(x)+z_{n}(x)
$$

where

$$
\left\{\begin{array}{c}
R_{n}^{\prime}(x)=-R_{n}^{2}(x)-\frac{1}{\alpha^{2}}\left[\beta_{n}+2 \frac{s_{n}-s_{n}-1}{\Delta t} \frac{\ell-x}{\ell-s_{n}}\right] R_{n}(x)+\frac{1}{\alpha^{2 \Delta t}}, R_{n}(\ell)=-\beta_{n} / \alpha^{2} \\
z_{n}^{\prime}(x)=-\left[R_{n}(x)+\frac{1}{\alpha^{2}}\left(\beta_{n}+2 \frac{s_{n}-s_{n-1}}{\Delta t} \frac{\ell-x}{\ell-s_{n}}\right)\right] z_{n}(x)-\frac{v_{n-1}(\vartheta(x))}{\alpha^{2 \Delta t}}, z_{n}(\ell)=q \beta_{n} / \alpha^{2}
\end{array}\right.
$$

Observe that by defining

$$
p(x)=R_{n}(x)+\frac{1}{\alpha^{2}}\left[\beta_{n}+2 \frac{s_{n}-s_{n-1}}{\Delta t} \frac{\ell-x}{\ell-s_{n}}\right],
$$

where

$$
R_{n}(x)=-\beta_{n} / \alpha^{2} \exp \left(\int_{x}^{\ell} p(\tau) d \tau\right)-1 /\left(\alpha^{2} \Delta t\right) \int_{x}^{\ell} \exp \left(\int_{x}^{s} p(\tau) d \tau\right) d s<0
$$

$v_{n}(x)$ can be determined from (16) with initial condition

$$
v_{n}\left(s_{n}\right)=\max \left\{0, \frac{Q\left(\beta_{n}\right)-z_{n}\left(s_{n}\right)}{-R_{n}\left(s_{n}\right)+a_{1}\left(\beta_{n}\right)}\right\}
$$

If $\pm v_{n}$ attains its maximum at $x_{0} \in\left(s_{n}, \ell\right)$, then 


$$
\pm v_{n}\left(x_{0}\right) \leq \pm v_{n-1}\left(\vartheta\left(x_{0}\right)\right)
$$

If $v_{n}$ attains its negative minimum at $x=\ell$, by the maximum principle (see [10]), $v_{n}^{\prime}(\ell)<0$, and hence $v_{n}(\ell)>q$ from the boundary condition at $x=\ell$. Therefore, we have a contradiction. If $v_{n}$ attains its positive maximum at $x=s_{n}$, then $v_{n}^{\prime}\left(s_{n}\right)<0$ and hence $v_{n}\left(s_{n}\right)=0$ by the boundary condition at $x=s_{n}$. Therefore, we obtain a contradiction. If $v_{n}$ attains its positive maximum at $x=\ell$, by the maximum principle, $v_{n}^{\prime}(\ell)>0$, and hence $v_{n}(\ell)<q$ from the boundary condition at $x=\ell$. Notice that if $v_{n}\left(s_{n}\right) \geq 0$ and $0 \leq v_{0}(x) \leq q$, then $0 \leq v_{n}(x) \leq q$. Next, let $\phi_{n}(x)=v_{n}^{\prime}(x)$. Then

$$
\left\{\begin{array}{c}
\alpha^{2} \phi_{n}^{\prime \prime}(x)+\left[\beta_{n}+2 \frac{s_{n}-s_{n-1}}{\Delta t} \frac{\ell-x}{\ell-s_{n}}\right] \phi_{n}^{\prime}(x)-\left[\frac{1}{\Delta t}+2 \frac{s_{n}-s_{n-1}}{\Delta t} \frac{1}{\ell-s_{n}}\right] \phi_{n}(x) \\
=\frac{-\phi_{n-1}(\vartheta(x))}{\Delta t} \frac{\ell-s_{n-1}}{\ell-s_{n}} \\
0 \leq \alpha^{2} \phi_{n}(\ell)=\beta_{n}\left(q-v_{n}(\ell)\right) \leq \beta_{M} q \\
0 \leq \phi_{n}\left(s_{n}\right) \leq a_{1}\left(\beta_{n}\right) v_{n}\left(s_{n}\right)+Q\left(\beta_{n}\right) \leq q\left\|a_{1}\right\|+\|Q\| .
\end{array}\right.
$$

The second inequality of (15) can be obtained by a direct application of the maximum principle for elliptic ordinary differential equations (see [10]). The proof is complete.

Next, take a family of nonpositive functions $\beta_{\epsilon} \in C^{2}(-\infty, \infty)$ with $\beta_{\epsilon}^{\prime} \leq 0, \epsilon>0$, and

$$
\beta_{\epsilon}(t)=\left\{\begin{array}{cc}
0, & \text { if } t \geq 0, \\
(t+\epsilon) / \epsilon, & \text { if } t<-2 \epsilon,
\end{array}\right.
$$

and consider a penalized problem of (14):

$$
\left\{\begin{aligned}
\frac{v_{\epsilon n}(x)-v_{\epsilon(n-1)}(\vartheta(x))}{\Delta t}= & \alpha^{2} v_{\epsilon n}^{\prime \prime}(x)+\left[\beta_{n}+2 \frac{s_{n}-s_{n-1}}{\Delta t} \frac{\ell-x}{\ell-s_{n}}\right] v_{\epsilon n}^{\prime}(x), s_{n}<x<\ell, \\
& \alpha^{2} v_{\epsilon n}^{\prime}(\ell)+\beta_{n} v_{\epsilon n}(\ell)=q \beta_{n} \\
-v_{\epsilon n}^{\prime}\left(s_{n}\right)+ & a_{1}\left(\beta_{n}\right) v_{\epsilon n}\left(s_{n}\right)+Q\left(\beta_{n}\right)+\beta_{\epsilon}\left(v_{\epsilon n}\left(s_{n}\right)\right)=0 \\
v_{\epsilon 0}(x)= & v_{0}(x), \vartheta(x)=\frac{\ell-s_{n-1}}{\ell-s_{n}} x-\frac{s_{n}-s_{n-1}}{\ell-s_{n}} \ell .
\end{aligned}\right.
$$

Note: $\quad \vartheta\left(s_{n}\right)=s_{n-1}, \vartheta(\ell)=\ell$.

Lemma 2: There exists a unique solution to (20) and the following estimates hold:

(i) $\quad-c_{0} \epsilon \leq v_{\epsilon n}(x) \leq q, c_{0}=\max \{2,1+\|Q\|\}$;

(ii) $\quad c_{1} \leq v_{\epsilon n}^{\prime}(x) \leq c_{2}$ 


$$
\begin{aligned}
& c_{1}=\max \left\{\min v_{0}^{\prime}(x),\left(c_{0}\left\|a_{1}\right\|+1-c_{0}\right) \epsilon\right\}, \\
& c_{2}=\max \left\{q\left\|a_{1}\right\|+\|Q\|,\left(q \beta_{M}+c_{0} \epsilon\right) / \alpha^{2}, \max v_{0}^{\prime}(x)\right\} ; \\
& \left(\text { iii) } \quad \mid v_{\epsilon n}(x)-v_{\epsilon(n-1)}(x)+\leq C \Delta t,\left\|v_{\epsilon n}^{\prime \prime}\right\| \leq C,\right.
\end{aligned}
$$

where $C$ is a constant depending only on the $C^{1}$ norm of $\beta(t), s(t)$ and $\ell-s(T)$, and the $C^{2}$ norm of $v_{0}$.

Proof: Again, we solve Equation (20) by the method of invariant imbedding. The solution is related by the Riccati transformation:

$$
v_{\epsilon n}^{\prime}(x)=R_{n}(x) v_{\epsilon n}(x)+z_{n}(x) .
$$

Substituting $v_{\epsilon n}^{\prime}(x)=R_{n}(x) v_{\epsilon n}(x)+z_{n}(x)$ into the boundary condition of (20) at $s_{n}$, we obtain

$$
\left(-R_{n}\left(s_{n}\right)+a_{1}\left(\beta_{n}\right)\right) v_{\epsilon n}\left(s_{n}\right)-z_{n}\left(s_{n}\right)+Q\left(\beta_{n}\right)+\beta_{\epsilon}\left(v_{\epsilon n}\left(s_{n}\right)\right)=0 .
$$

Define

$$
f(t)=\left(-R_{n}\left(s_{n}\right)+a_{1}\left(\beta_{n}\right)\right) t-z_{n}\left(s_{n}\right)+Q\left(\beta_{n}\right)+\beta_{\epsilon}(t)
$$

Then

$$
\begin{gathered}
f^{\prime}(t)=\left(-R_{n}\left(s_{n}\right)+a_{1}\left(\beta_{n}\right)\right)+\beta_{\epsilon}^{\prime}(t)>0, \\
f(+\infty)=\infty, f(-\infty)=-\infty
\end{gathered}
$$

and hence there exists a unique $t_{0}$ such that $f\left(t_{0}\right)=0$. Therefore, Equation (20) has a unique solution with $v_{\epsilon n}\left(s_{n}\right)=t_{0}$.

Now, we shall use mathematical induction. For $n=0$, the conclusions $(i)$ and $(i i)$ hold. Suppose that it is correct for $k<n$. We shall consider the case of $k=n$. By the maximum principle from [10], if $v_{\epsilon n}$ attains its negative minimum at $x_{0}$, then either $v_{\epsilon n}\left(x_{0}\right) \geq v_{\epsilon(n-1)}\left(\vartheta\left(x_{0}\right)\right)$ or $Q\left(\beta_{n}\right)+\beta_{\epsilon}\left(v_{\epsilon n}\left(x_{0}\right)\right) \geq 0$ depending on whether $x_{0}$ lies on the interior of $\left(s_{n}, \ell\right)$ or on its boundaries. So

$$
\begin{aligned}
& \text { either } v_{\epsilon n}(x) \geq-2 \epsilon \\
& \text { or } v_{\epsilon n}(x) \geq-\left[1+Q\left(\beta_{n}\right)\right] \epsilon \geq-[1+\|Q\|] \epsilon,
\end{aligned}
$$

since $v_{0}(x) \geq 0$. This is the first part of $(i)$. If $v_{\epsilon n}$ attains its positive maximum at $x_{0}$, then $v_{\epsilon n}\left(x_{0}\right) \leq v_{\epsilon(n-1)}\left(\vartheta\left(x_{0}\right)\right), v_{\epsilon n}\left(x_{0}\right) \leq q$, or $v_{\epsilon n}\left(x_{0}\right) \leq 0$. This is the second part of $(i)$. To prove (ii), let $\psi_{n}(x)=v_{\epsilon n}^{\prime}(x)$. Then 


$$
\left\{\begin{array}{c}
\alpha^{2} \psi_{n}^{\prime \prime}(x)+\left[\beta_{n}+2 \frac{s_{n}-s_{n-1}}{\Delta t} \frac{\ell-x}{\ell-s_{n}}\right] \psi_{n}^{\prime}(x)-\left[\frac{1}{\Delta t}+2 \frac{s_{n}-s_{n-1}}{\Delta t} \frac{1}{\ell-s_{n}}\right] \psi_{n}(x) \\
=\frac{-\psi_{(n-1)}(\vartheta(x))}{\Delta t} \frac{\ell-s_{n-1}}{\ell-s_{n}}, \\
\alpha^{2} \psi_{n}(\ell)=\beta_{n}\left(q-v_{\epsilon n}(\ell)\right), \\
\psi_{n}\left(s_{n}\right)=a_{1}\left(\beta_{n}\right) v_{\epsilon n}\left(s_{n}\right)+Q\left(\beta_{n}\right)+\beta_{\epsilon}\left(v_{\epsilon n}\left(s_{n}\right)\right) .
\end{array}\right.
$$

Using a similar argument as above, we get the second part of (ii). Now, we shall prove (iii). For this purpose, let

$$
\eta_{n}(x)= \pm v_{\epsilon n}^{\prime \prime}(x) e^{-\chi(x-\ell-\delta)^{2}}, \delta=\frac{\ell-s_{n}}{2}, \chi>0 .
$$

Then $\eta_{n}(x)$ satisfies the following equation:

$$
\begin{gathered}
\alpha^{2} \eta_{n}^{\prime \prime}(x)+\left[4 \chi \alpha^{2}(x-\ell+\delta)+\beta_{n}+2 \frac{s_{n}-s_{n}-1}{\Delta t} \frac{\ell-x}{\ell-s_{n}}\right] \eta_{n}^{\prime}(x)-\left[\frac{1}{\Delta t}-2 \chi \alpha^{2}\right. \\
\left.-4 \chi^{2} \alpha^{2}(x-\ell+\delta)^{2}-2 \chi(x-\ell+\delta)\left(\beta_{n}+2 \frac{s_{n}-s_{n-1}-1}{\Delta t} \frac{\ell-x}{\ell s_{n}}\right)+4 \frac{s_{n}-s_{n-1}}{\Delta t} \frac{1}{\ell-s_{n}}\right] \eta_{n}(x) \\
=\mp \frac{1}{\Delta t} \eta_{n-1}(\vartheta(x)) \pm \eta_{n-1}(\vartheta(x)) K(x),
\end{gathered}
$$

where

$$
K(x)=1-e^{\chi(\vartheta(x)-\ell-\delta)^{2}-\chi(x-\ell-\delta)^{2}}\left(\frac{\ell-s_{n}-1}{\ell-s_{n}}\right)
$$

and the following boundary conditions:

$$
\begin{gathered}
\alpha^{2} \eta_{n}^{\prime}(\ell)+\left(\beta_{n}+\beta_{n-1}+2 \chi \delta \alpha^{2}\right) \eta_{n}(\ell) \\
= \pm\left[\left[2 \frac{s_{n}-s_{n-1}}{\Delta t} \frac{1}{\ell-s_{n}}-\beta_{n} \beta_{n-1} / \alpha^{2}\right] v_{\epsilon n}^{\prime}(\ell)-\frac{s_{n}-s_{n-1}}{\Delta t} \frac{1}{\ell-s_{n}} v_{\epsilon(n-1)}^{\prime}(\ell)\right. \\
\left.\quad+\frac{\beta_{n}-\beta_{n-1}}{\Delta t}\left(q-v_{\epsilon n}(\ell)\right) / \alpha^{2}\right] e^{-\chi \delta^{2}}=K_{2}(\epsilon), \\
\alpha^{2} \eta_{n}^{\prime}\left(s_{n}\right)-\left[2 \chi \alpha^{2}\left(\ell-s_{n}-\delta\right)+\alpha^{2} \beta_{e}^{\prime}(\xi)+\alpha^{2} a_{1}\left(\beta_{n-1}\right)-\beta_{n}-2 \frac{s_{n}-s_{n-1}}{\Delta t}\right] \eta_{n}\left(s_{n}\right) \\
= \pm\left[\left[\frac{s_{n}-s_{n-1}}{\Delta t} \frac{1}{\ell-s_{n}}+\left(a_{1}\left(\beta_{n-1}\right)+\beta_{\epsilon}^{\prime}(\xi)\right)\left(\beta_{n}+2 \frac{s_{n}-s_{n-1}}{\Delta t}\right)\right] v_{\epsilon n}^{\prime}\left(s_{n}\right)\right. \\
+\left(\beta_{\epsilon}^{\prime}(\vartheta)+a_{1}\left(\beta_{n}\right)+\frac{s_{n}-s_{n-1}}{\Delta t} \frac{1}{\ell-s_{n}}\right) v_{\epsilon n}^{\prime}\left(s_{n}\right)-\frac{s_{n}-s_{n-1}}{\Delta t} \frac{1}{\ell-s_{n}} v_{\epsilon(n-1)}^{\prime}\left(s_{n-1}\right) \\
\left.+\frac{a_{1}\left(\beta_{n}\right)-a_{1}\left(\beta_{n-1}\right)}{\Delta t} v_{\epsilon n}\left(s_{n}\right)+\frac{Q\left(\beta_{n}\right)-Q\left(\beta_{n-1}\right)}{\Delta t}\right] e^{-\chi\left(s_{n}-\ell-\delta\right)^{2}}=K_{3}(\epsilon),
\end{gathered}
$$

where $-c_{0} \epsilon \leq \xi \leq q$ and $\chi>0$ is such that

$$
2 \chi \delta \alpha^{2} \geq 1,2 \chi \alpha^{2}\left(\ell-s_{n}-\delta\right)-\beta_{n}-2 \frac{s_{n}-s_{n}-1}{\Delta t} \geq 1
$$


Suppose that $\eta_{n-1}(x) \leq C_{n-1}$. Then if $\eta_{n}$ attains its maximum in the interior of $\left(s_{n}, \ell\right)$, then by the inequality $|K(x)| \leq K_{0} \Delta t$, we obtain

$$
\eta_{n}(x) \leq C_{n-1}+K_{10} \Delta t C_{n-1}, \text { where } K_{10}>0 \text { is a constant }
$$

and hence

$$
\eta_{n}(x) \leq\left(1+K_{10} \Delta t\right)^{N} C_{0}, \Delta t=T / N
$$

If $\eta_{n}(x)$ attains its maximum at $x=\ell$ or $x=s_{n}$ then by $(i)$ and $(i i)$,

$$
\eta_{n}(x) \leq K_{2}(\epsilon) \leq K_{20}, \quad \eta_{n}(x) \leq K_{3}(\epsilon) \leq K_{30}
$$

Since

$$
\lim _{t \rightarrow \infty}\left(1+K_{10} \Delta t\right)^{N}=K_{10} T
$$

we, finally, get from (20) that $\eta_{n}(x) \leq C$ or $\left|v_{\epsilon n}^{\prime \prime}\right| \leq C$. Since

$$
\begin{gathered}
\frac{v_{\epsilon n}(x)-v_{\epsilon(n-1)}(x)}{\Delta t}=\alpha^{2} v_{\epsilon n}^{\prime \prime}(x)+\left[\beta_{n}+2 \frac{s_{n}-s_{n}-1}{\Delta t} \frac{\ell-x}{\ell-s_{n}}\right] v_{\epsilon n}^{\prime}(x)-v_{\epsilon(n-1)}^{\prime}(\xi) \frac{x-\vartheta}{\Delta t}, \\
\xi \in(\vartheta(x), x), \frac{x-\vartheta}{\Delta t}=\frac{s_{n}-s_{n}-1}{\Delta t} \frac{\ell-x}{\ell-s_{n}}
\end{gathered}
$$

we have $\left|\frac{v_{\epsilon n}(x)-v_{\epsilon(n-1)}(x)}{\Delta t}\right| \leq C$. The proof is complete.

Since the constant $C$ in Lemma 2 is independent on $\epsilon$, by the Ascoli-Arzela theorem applied for any integer $n$, there exists a subsequence of $\left\{v_{\epsilon n}(x), v_{\epsilon n}^{\prime}(x)\right\}$, which for easier notation is still denoted by $v_{\epsilon n}$, such that

$$
v_{\epsilon n} \rightarrow v_{n}, v_{\epsilon n}^{\prime} \rightarrow v_{n}^{\prime} \text {, uniformly as } \epsilon \rightarrow 0 \text {, }
$$

and, from equation (20),

$$
v_{\epsilon n}^{\prime \prime} \rightarrow v_{n}^{\prime \prime} \text {, uniformly as } \epsilon \rightarrow 0 \text {. }
$$

If $v_{n}\left(x_{0}\right)>0$, then by uniform convergence, for sufficiently small $\epsilon$, there exists a constant $c>0$ such that $v_{\epsilon n}\left(x_{0}\right) \geq c>0$. Therefore, from $(20),-v_{\epsilon n}^{\prime}\left(s_{n}\right)+a_{1}\left(\beta_{n}\right) v_{\epsilon n}\left(s_{n}\right)+Q\left(\beta_{n}\right)=0$, and hence $-v_{n}^{\prime}\left(s_{n}\right)+a_{1}\left(\beta_{n}\right) v_{n}\left(s_{n}\right)+Q\left(\beta_{n}\right)=0$, i.e. $v_{n}(x)$ is the solution of (14). Since the solution of (14) is unique, the whole sequence of solutions of (20) converges uniformly to the solution of (14) as $\epsilon$ goes to zero.

We thus have proved the following lemma. 
Lemma 3: The solution of Equation (14) satisfies the inequality

$$
\left|v_{n}(x)-v_{n-1}(x)\right| \leq C \Delta t, \quad\left\|v_{n}^{\prime \prime}\right\| \leq C
$$

where $C$ is a constant depending only on the $C^{1}$ norm of $\beta(t), s(t), \ell-s(T)$, and the $C^{2}$ norm of $v_{0}$.

Define

$$
\left\{\begin{array}{c}
s_{N}(t)=\frac{1}{\Delta t}\left[\left(t-t_{n-1}\right) s_{n}+\left(t_{n}-t\right) s_{n-1}\right] \text { for } t \in\left[t_{n-1}, t_{n}\right), \\
v_{N}(x, t)=\frac{1}{\Delta t}\left[\left(t-t_{n-1}\right) v_{n}(x)+\left(t_{n}-t\right) v_{n-1}(x)\right] \text { for } t \in\left[t_{n-1}, t_{n}\right) .
\end{array}\right.
$$

\section{Lemma 4:}

(a) $0 \leq s_{N}^{\prime}(t) \leq\left\|s^{\prime}\right\|$;

(b) $\quad 0 \leq v_{N}(x, t) \leq q$;

(c) $\left|v_{N x}(x, t)\right| \leq \max \left\{\left\|v_{0}\right\|, q \beta_{M} / \alpha^{2}, q\left\|a_{1}\right\|+\|Q\|\right\}$;

(d) $\quad v_{N x}(x, t) \geq 0$, if $u_{0}^{\prime}(x) \leq 0$;

(e) $\quad\left|v_{N x x}(x, t)\right| \leq C$;

(f) $\quad\left|v_{N x}\left(x, t_{1}\right)\right|=\left|v_{N x}\left(x, t_{2}\right)\right| \leq C\left|t_{1}-t_{2}\right|^{1 / 2}$ for any $t_{1}, t_{2} \in\left[t_{n-1}, t_{n}\right)$,

where $C$ has the same meaning as in Lemma 3.

Proof: Conditions (a)-(e) are direct consequences of Lemma 1 and Lemma 3, and the definition of $v_{N}(x, t)$. Now, we shall only consider condition $(f)$. Notice that

$$
v_{N x t}(x, t)=\frac{v_{n}^{\prime}(x)-v_{n-1}^{\prime}(x)}{\Delta t}
$$

and

$$
\begin{gathered}
v_{N x t}(x, t)=\alpha^{2} v_{n}^{\prime \prime \prime}(x)+\left[\beta_{n}+2 \frac{s_{n}-s_{n}-1}{\Delta t} \frac{\ell-x}{\ell-s_{n}}\right] v_{n}^{\prime \prime}(x)-\left[2 \frac{s_{n}-s_{n-1}}{\Delta t} \frac{1}{\ell-s_{n}}\right] v_{n}^{\prime}(x) \\
-v_{n-1}^{\prime}(\xi) \frac{s_{n}-s_{n-1}}{\Delta t} \frac{\ell-x}{\ell-s_{n}}=\alpha^{2} v_{n}^{\prime \prime \prime}(x)+G_{n}(x),
\end{gathered}
$$

where $\quad\left|G_{n}(x)\right| \leq C, \quad \xi \in(\vartheta(x), x), \quad t \in\left[t_{n-1}, t_{n}\right) . \quad$ For $\quad$ any $\quad t_{n-1} \leq t_{1}<t_{2}<t_{n}$, $\tau=\left(t_{2}-t_{1}\right)^{1 / 2}$

$$
\begin{gathered}
\int_{x}^{x+\tau}\left[v_{N x}\left(y, t_{1}\right)-v_{N x}\left(y, t_{2}\right)\right] d y=\int_{x}^{x+\tau} \int_{t_{1}}^{t_{2}} v_{N x t}(y, t) d y d t \\
=\left[\alpha^{2}\left[v_{n}^{\prime \prime}(x+\tau)-v_{n}^{\prime \prime}(x)\right]+\int_{x}^{x+\tau} G_{n}(y) d y\right]\left(t_{2}-t_{1}\right) .
\end{gathered}
$$


Hence there exists a point $\xi \in(x, x+\tau)$ such that

$$
\left|v_{N x}\left(\xi, t_{1}\right)-v_{N x}\left(\xi, t_{2}\right)\right| \leq C\left|t_{1}-t_{2}\right|^{1 / 2}
$$

Thus

$$
\begin{gathered}
\left|v_{N x}\left(x, t_{1}\right)-v_{N x}\left(x, t_{2}\right)\right| \leq\left|v_{N x}\left(x, t_{1}\right)-v_{N x}\left(\xi, t_{1}\right)\right| \\
+\left|v_{N x}\left(x, t_{2}\right)-v_{N x}\left(\xi, t_{2}\right)\right|+C\left|t_{1}-t_{2}\right|^{1 / 2} \leq\left(C+2\left\|v_{N x x}\right\|\right)\left|t_{1}-t_{2}\right|^{1 / 2}
\end{gathered}
$$

and consequently $(f)$ holds. The proof is complete.

Since $s^{\prime}(t)$ is a continuous function on $[0, T]$,

$$
\lim _{N \rightarrow \infty}\left\|s_{N}-s\right\|=0, \lim _{N \rightarrow \infty} \max _{n}\left\|\frac{s_{n}-s_{n}-1}{\Delta t}-s^{\prime}(t)\right\|_{c\left[t_{n-1}, t_{n}\right]}=0 .
$$

By the Ascoli-Arzela theorem, there exist subsequences of $v_{N}(x, t), s_{N}(t)$, which for easier notation are still denoted by $v_{N}, s_{N}$, and a function $\phi(x, t)$ such that

$$
\begin{gathered}
s_{N} \rightarrow s, v_{N} \rightarrow \phi, v_{N x} \rightarrow \phi_{x} \text { uniformly as } N \rightarrow \infty, \\
v_{N t} \rightarrow \phi_{t}, v_{N x x} \rightarrow \phi_{x x} \text { in } L^{\infty} \text { weak star topology, }
\end{gathered}
$$

and

$$
\begin{gathered}
0 \leq \phi \leq q,\left|\phi_{x}(x, t)\right| \leq C_{0},\left|\phi_{t}(x, t)\right| \leq C, \mid \phi_{x x}(x, t) \leq C, \\
\left|\phi_{x}\left(x, t_{1}\right)\right|-\left|\phi_{x}\left(x, t_{2}\right)\right| \leq C\left|t_{1}-t_{2}\right|^{1 / 2}, \text { for any } t_{1}, t_{2} \in[0, T]
\end{gathered}
$$

where $C$ is the constant from Lemma 4 .

Notice that

$$
\begin{gathered}
-v_{N}^{\prime}\left(s_{N}\left(t_{n}\right), t_{n}\right)+a_{1}\left(\beta\left(t_{n}\right)\right) v_{N}\left(s_{N}\left(t_{n}\right), t_{n}\right)+Q\left(\beta\left(t_{n}\right)\right) \geq 0, \\
{\left[v_{N}\left(s_{N}\left(t_{n}\right), t_{n}\right)\right]\left[-v_{N}^{\prime}\left(s_{N}\left(t_{n}\right), t_{n}\right)+a_{1}\left(\beta\left(t_{n}\right)\right) v_{N}\left(s_{N}\left(t_{n}\right), t_{n}\right)+Q\left(\beta\left(t_{n}\right)\right)\right]=0,} \\
\alpha^{2} v_{N}^{\prime}\left(\ell, t_{n}\right)+\beta\left(t_{n}\right) v_{N}\left(\ell, t_{n}\right)=q \beta\left(t_{n}\right),
\end{gathered}
$$

at each point of the partition $\left\{t_{n}\right\}$ associated with $N$. Since these points are dense in $[0, T]$ as $N \rightarrow \infty$ and the convergence of $v_{N x}(x, t), v_{N}(x, t), s_{N}(t)$ is uniform, it follows immediately that

$$
\left\{\begin{array}{c}
-\phi_{x}(s(t), t)+a_{1}(\beta(t)) \phi(s(t), t)+Q(\beta(t)) \geq 0, \\
\phi(s(t), t)\left[-\phi^{\prime}(s(t), t)+a_{1}(\beta(t)) \phi(s(t), t)+Q(\beta(t)]=0,\right. \\
\alpha^{2} \phi_{x}(\ell, t)+\beta(t) \phi(\ell, t)=q \beta(t) .
\end{array}\right.
$$

We shall show that $\phi(x, t)=v(x, t)$ is the solution of fixed problem (8). In fact, since 


$$
\begin{gathered}
v_{N t}(x, t)=\alpha^{2} v_{n}^{\prime \prime}(x)+\left[\beta_{n}+\frac{s_{n}-s_{n-1}}{\Delta t} \frac{\ell-x}{\ell-s_{n}}\right] v_{n}^{\prime}(x)+K_{n}(x) \\
=\alpha^{2} v_{N x x}\left(x, t_{n}\right)+\left[\beta\left(t_{n}\right)+s_{N}^{\prime}(t) \frac{\ell-x}{\ell-s_{N}\left(t_{n}\right)}\right] v_{N x}\left(x, t_{n}\right)+K_{n}(x), \\
s_{N}\left(t_{n}\right)<x<\ell, t \in\left(t_{n-1}, t_{n}\right), n \geq 1
\end{gathered}
$$

where

$$
\begin{gathered}
K_{n}(x)=\left[v_{n}^{\prime}(x)-v_{n-1}^{\prime}(\xi)\right] \frac{s_{n}-s_{n-1}}{\Delta t} \frac{\ell-x}{\ell-s_{n}}=\left[v_{n}^{\prime}(x)-v_{n-1}^{\prime}(x)\right] \frac{s_{n}-s_{n-1}}{\Delta t} \frac{\ell-x}{\ell-s_{n}} \\
+v_{n-1}^{\prime \prime}(\Theta) \frac{s_{n}-s_{n-1}}{\Delta t} \frac{\ell-x}{\ell-s_{n}}(x-\xi), \xi, \Theta \in(\vartheta(x), x), \frac{x-\vartheta}{\Delta t}=\frac{s_{n}-s_{n-1}}{\Delta t} \frac{\ell-x}{\ell-s_{n}} \\
\left|K_{n}(x)\right| \leq C \Delta t^{1 / 2} .
\end{gathered}
$$

For any $f(x, t) \in C^{2}(\Omega), \Omega=\{(x, t), t \in[0, T], x \in[s(t), \ell]\}$,

where

$$
\begin{aligned}
& \sum_{n=1}^{N} \Delta t \int_{s_{N}\left(t_{n}\right)}^{\ell} v_{N t}(x, t) f\left(x, t_{n}\right) d x=\sum_{n=1}^{N} \Delta t \int_{s_{N}\left(t_{n}\right)}^{\ell} \alpha^{2} v_{N x x}\left(x, t_{n}\right) f\left(x, t_{n}\right) d x \\
& +\sum_{n=1}^{N} \Delta t \int_{s_{N}\left(t_{n}\right)}^{\ell}\left[\beta\left(t_{n}\right)+s_{N}^{\prime}\left(t_{n}\right) \frac{\ell-x}{\ell-s_{N}\left(t_{n}\right)}\right] v_{N x}\left(x, t_{n}\right) f\left(x, t_{n}\right) d x+G_{N}
\end{aligned}
$$

$$
\begin{gathered}
\left|G_{N}\right| \leq\left|\sum_{n=1}^{N} \Delta t \int_{s_{N}\left(t_{n}\right)}^{\ell} K_{n}(x) f\left(x, t_{n}\right) d x\right| \leq C T(\ell-s(T))\|f\| \Delta t^{1 / 2}, \\
\lim _{N \rightarrow \infty} G_{N}=0 .
\end{gathered}
$$

By using integration by parts and by uniform convergence of (25) and (26), we can obtain

$$
\begin{gathered}
-\iint_{\Omega} f_{t} \phi d x d t+\int_{0}^{T} s^{\prime}(t)[\phi f](s(t), t) d t+\int_{s(T)}^{\ell}[f \phi](x, T) d x-\int_{0}^{\ell} f(x, 0) v_{0}(x) d x \\
=\alpha^{2} \int_{0}^{T}\left[\left[\phi_{x} f\right](l, t)-\left[\phi_{x} f\right](s(t), t)\right] d t-\alpha^{2} \iint_{\Omega} \phi_{x} f_{x} d x d t \\
+\iint_{\Omega}\left[\beta(t)+s^{\prime}(t) \frac{\ell-x}{\ell-s(t)}\right] \phi_{x} f d x d t
\end{gathered}
$$

and (28) holds for any continuous function $f$ with first order derivatives bounded on $\Omega$ by smooth function approximation.

From Equation (8) it also holds for its solution $v$ that 


$$
\begin{gathered}
-\iint_{\Omega} f_{t} v d x d t+\int_{0}^{T} s^{\prime}(t)[v f](s(t), t) d t+\int_{s(T)}^{\ell}[f v](x, T) d x-\int_{0}^{\ell} f(x, 0) v_{0}(x) d x \\
=\alpha^{2} \int_{0}^{T}\left[\left[v_{x} f\right](l, t)-\left[v_{x} f\right](s(t), t)\right] d t-\alpha^{2} \iint_{\Omega} v_{x} f_{x} d x d t \\
+\iint_{\Omega}\left[\beta(t)+s^{\prime}(t) \frac{\ell-x}{\ell-s(t)}\right] v_{x} f d x d t .
\end{gathered}
$$

Let $\delta>\left\|\frac{s^{\prime}(t)}{\ell-s(t)}\right\|, f=(v-\phi) e^{-\delta t}$ in $(28)$ and $f=(\phi-v) e^{-\delta t}$ in (29). After these substitutions, and then adding (28) and (29), we obtain

$$
\begin{gathered}
\alpha^{2} \iint_{\Omega}(v-\phi)_{x}^{2} e^{-\delta t} d x d t+1 / 2 e^{-\delta T} \int_{s(T)}^{\ell}(v-\phi)^{2}(x, T) d x \\
+1 / 2 \int_{0}^{T} \beta(t)(v-\phi)^{2}(\ell, t) e^{-\delta t} d t+\int_{0}^{T} s^{\prime}(t)(v-\phi)^{2}(s(t), t) e^{-\delta t} d t \\
+1 / 2 \int_{0}^{T} \beta(t)(v-\phi)^{2}(s(t), t) e^{-\delta t} d t+1 / 2 \iint_{\Omega}\left(\delta-\frac{s^{\prime}(t)}{\ell-s(t)}\right)(v-\phi)^{2} e^{-\delta t} d x d t \\
=-\alpha^{2} \int_{0}^{T}(v-\phi)(v-\phi)_{x}(s(t), t) d t \leq 0 .
\end{gathered}
$$

The last inequality comes from the fact that

$$
(v-\phi)(v-\phi)_{x}(s(t), t) d t \leq 0
$$

by the condition at $x=s(t)$. Hence $\phi=v$. This demonstration implies that the (weak) solution of fixed problem (8) is unique and, hence, that (26) holds for the whole sequence $v_{N}$. Thus we have proved the following:

Theorem 1: Let $s_{N}(t)$ and $v_{N}(x, t)$ be defined as in (24), where $v_{n}(x)$ is produced by the Rothe approximation scheme (14) for fixed problem (8). Then

$$
\begin{gathered}
v_{N} \rightarrow v, v_{N x} \rightarrow v_{x} \text { uniformly as } N \rightarrow \infty, \\
v_{N t} \rightarrow v_{t}, v_{N x x} \rightarrow v_{x x} \text { in } L^{\infty} \text { weak star topology, }
\end{gathered}
$$

where $v$ is the unique solution of Equation (8), and $v$ satisfies

$$
0 \leq v \leq q,\left|v_{x}(x, t)\right| \leq C_{0},\left|v_{t}(x, t)\right| \leq C,\left|v_{x x}(x, t)\right| \leq C,
$$




$$
\left|v_{x}\left(x, t_{1}\right)\right|-\left|v_{x}\left(x, t_{2}\right)\right| \leq C\left|t_{1}-t_{2}\right|^{1 / 2} \text { for any } t_{1}, t_{2} \in[0, T] \text {, }
$$

where $C$ depends only on the $C^{1}$ norm of $\beta, s, \ell-s(T)$, and the $C^{2}$ norm of $v_{0}$.

\section{EXISTENCE OF THE SOLUTION}

This section will contribute to the existence of solution of Equations (8)-(9). For this purpose, we construct the Rothe approximation scheme of Equations (8)-(9) as follows:

$$
\left\{\begin{array}{c}
\frac{v_{n}(x)-v_{n-1}(\vartheta(x))}{\Delta t}=\alpha^{2} v_{n}^{\prime \prime}(x)+\left[\beta_{n}+2 \frac{s_{n}-s_{n-1}}{\Delta t} \frac{\ell-x}{\ell-s_{n}}\right] v_{n}^{\prime}(x), s_{n}<x<\ell, \\
\alpha^{2} v_{n}^{\prime}(\ell)+\beta_{n} v_{n}(\ell)=q \beta_{n}, \\
v_{n}\left(s_{n}\right) \geq 0,-v_{n}^{\prime}\left(s_{n}\right)+a_{1}\left(\beta_{n}\right) v_{n}\left(s_{n}\right)+Q\left(\beta_{n}\right) \geq 0, \\
v_{n}\left(s_{n}\right)\left[-v_{n}^{\prime}\left(s_{n}\right)+a_{1}\left(\beta_{n}\right) v_{n}\left(s_{n}\right)+Q\left(\beta_{n}\right)\right]=0, n=1,2, \ldots, N, \\
v_{0}(x)=v_{m}-v_{0}(x), \vartheta(x)=\frac{\ell-s_{n-1}}{\ell-s_{n}} x-\frac{s_{n}-s_{n-1}}{\ell-s_{n}} \ell, \\
s_{n}=s_{n-1}+\frac{\kappa}{\rho L}\left[-v_{n-1}^{\prime}\left(s_{n-1}\right)+a_{1}\left(\beta_{n-1}\right) v_{n-1}\left(s_{n-1}\right)+Q\left(\beta_{n-1}\right)\right] \Delta t, s_{0}=0,
\end{array}\right.
$$

and $s_{N}(t), v_{N}(x, t)$ are defined in the same form as in (24) but $s_{n}$ and $v_{n}$ are produced by (30).

Arguing as in Lemma 1-4, we have

Lemma 5: There exists a unique solution to Equation (30) and the corresponding functions $s_{N}(t)$ and $v_{N}(x, t)$ satisfy conditions $(b)-(f)$ of Lemma 4. Moreover,

$$
0 \leq s_{N}^{\prime}(t)=C_{0} \leq 2 \frac{\kappa}{\rho L} \max \left\{\left\|v_{0}\right\|, q \beta_{M} / \alpha^{2}, q\left\|a_{1}\right\|+\|Q\|\right\}
$$

Now

$$
\begin{gathered}
s_{N}(T)=s_{N}=\sum_{n=1}^{N}\left(s_{n}-s_{n-1}\right) \\
=\frac{\kappa}{\rho L} \sum_{n=1}^{N}\left[-v_{n-1}^{\prime}\left(s_{n-1}\right)+a_{1}\left(\beta_{n-1}\right) v_{n-1}\left(s_{n-1}\right)+Q\left(\beta_{n-1}\right)\right] \Delta t \leq C_{0} T .
\end{gathered}
$$

If $T$ is sufficiently small, then $s_{N}<$ const. $<\ell$ for all $N$. Let $\mathbb{S}(t)$ and $\Phi(x, t)$ be the uniform limits of subsequence of $s_{N}(t)$ and $v_{N}(x, t)$, which for easier notation are still denoted by $s_{N}$ and $v_{N}$, as $N$ goes to infinity. Observe that

$$
\frac{\rho L}{\kappa} s_{N}(T)=\sum_{n=1}^{N}\left(s_{n}-s_{n-1}\right)
$$




$$
\begin{gathered}
=\sum_{n=1}^{N}\left[-v_{n-1}^{\prime}\left(s_{n-1}\right)+a_{1}\left(\beta_{n-1}\right) v_{n-1}\left(s_{n-1}\right)+Q\left(\beta_{n-1}\right)\right] \Delta t \\
=\sum_{n=1}^{N}-v_{N x}\left(s_{N}\left(t_{n-1}\right), t_{n-1}\right) \Delta t+\sum_{n=1}^{N} a_{1}\left(\beta_{n-1}\right) v_{N}\left(s_{N}\left(t_{n-1}\right), t_{n-1}\right) \Delta t \\
\quad+\sum_{n=1}^{N} Q\left(\beta_{n-1}\right) \Delta t \\
=\sum_{n=1}^{N}\left[\Phi_{x}\left(s_{N}\left(t_{n-1}\right), t_{n-1}\right)-v_{N x}\left(s_{N}\left(t_{n-1}\right), t_{n-1}\right)\right] \Delta t \\
+\sum_{n=1}^{N}\left[-\Phi_{x}\left[s_{N}\left(t_{n-1}\right), t_{n-1}\right)+\Phi_{x}\left(\mathbb{S}\left(t_{n-1}\right), t_{n-1}\right)\right] \Delta t \\
+\sum_{n=1}^{N} a_{1}\left(\beta_{n-1}\right)\left[v_{N}\left(s_{N}\left(t_{n-1}\right), t_{n-1}\right)-\Phi\left(s_{N}\left(t_{n-1}\right), t_{n-1}\right)\right] \Delta t \\
+\sum_{n=1}^{N} a_{1}\left(\beta_{n-1}\right)\left[\Phi\left(s_{N}\left(t_{n-1}\right), t_{n-1}\right)-\Phi\left(\mathbb{S}\left(t_{n-1}\right), t_{n-1}\right)\right] \Delta t \\
+\sum_{n=1}^{N}\left[-\Phi_{x}\left(s\left(t_{n-1}\right), t_{n-1}\right)+a_{1}\left(\beta_{n-1}\right) \Phi\left(\mathbb{S}\left(t_{n-1}\right), t_{n-1}\right)+Q\left(\beta_{n-1}\right)\right] \Delta t .
\end{gathered}
$$

The last term of the above formula is a Riemann sum. From uniform convergence we have immediately that

$$
\mathbb{S}(T)=\frac{\kappa}{\rho L} \int_{0}^{T}\left[-\Phi_{x}(\mathbb{S}(t), t)+a_{1}(\beta(t)) \Phi(x, t)+Q(\beta(t))\right] d t .
$$

Considering $t$ as any $t \in(0, T]$ we also have

$$
\mathbb{S}(t)=\frac{\kappa}{\rho L} \int_{0}^{t}\left[-\Phi_{x}(\mathbb{S}(t), t)+a_{1}(\beta(t)) \Phi(x, t)+Q(\beta(t))\right] d t .
$$

From the same arguments in (26), we also have

$$
\left\{\begin{array}{c}
\Phi(\mathbb{S}(t), t) \geq 0,-\Phi^{\prime}(\mathbb{S}(t), t)+a_{1}(\beta(t)) \Phi(x, t)+Q(\beta(t)) \geq 0 \\
\Phi(\mathbb{S}(t), t)\left[-\Phi^{\prime}(\mathbb{S}(t), t)+a_{1}(\beta(t)) \Phi(x, t)+Q(\beta(t)]=0\right. \\
\alpha^{2} \Phi_{x}(\ell, t)+\beta(t) \Phi(\ell, t)=q \beta(t) .
\end{array}\right.
$$

From (32), $\mathrm{S}(t)$ is a $C^{1}$ function on $[0, T]$. Using the same argument as in the proof of Theorem 1, we can prove for $\$(t), \Phi(x, t)$ is a solution of Equations (8)-(9). Finally, it is easy to see that

$$
\left\|\Phi_{x x}\right\| \leq C,
$$


where $C$ depends only on the $C^{1}$ norm of $\beta$ and $s(t)$, the $C^{2}$ norm of $v_{0}(x)$, and $\ell-s(T)$. Since $\mathrm{S}^{\prime}(t) \leq C_{0}$, where $C_{0}$ is defined in (31),C only depends on the $C^{1}$ of $\beta$, the $C^{2}$ norm of $v_{0}(x)$, and $\ell-s(T)$. We can extend this solution until $S(T)=\ell$ (e.g. [12]).

\section{Theorem 2:}

(i) There exists a solution $(s(t), u(x, t)),(x, t) \in[s(t), \ell] \times[0, T]$ to Equation (4) and either $T=\infty, s(t)<\ell$ for all $t \geq 0$; or $T<\infty$ and $s(T)=\ell$.

This solution can be obtained as the uniform limit of the following Rothe approximation solutions $s_{N}(t)$ and $u_{N}(x, t)$ :

$$
\left\{\begin{array}{c}
s_{N}(t)=\frac{1}{\Delta t}\left[\left(t-t_{n-1}\right) s_{n}+\left(t_{n}-t\right) s_{n-1}\right], \text { for } t \in\left[t_{n-1}, t_{n}\right), \\
u_{N}(x, t)=\frac{1}{\Delta t}\left[\left(t-t_{n-1}\right) u_{n}(x)+\left(t_{n}-t\right) u_{n-1}(x)\right], \text { for } t \in\left[t_{n-1}, t_{n}\right)
\end{array}\right.
$$

in which $s_{n}$ and $v_{n}$ are produced by

$$
\left\{\begin{array}{c}
s_{n}=s_{n-1}+\frac{\kappa}{\rho L}\left[u_{n-1}^{\prime}\left(s_{n-1}\right)-a_{1}\left(\beta_{n-1}\right) v_{n-1}\left(s_{n-1}\right)+a_{2}\left(\beta_{n-1}\right)\right] \Delta t \\
u_{n}^{\prime}(x)=R_{n}(x) u_{n}(x)+z_{n}(x) \\
u_{n}\left(s_{n}\right)=\min \left\{u_{m}, \frac{a_{2}\left(\beta_{n}\right)+z_{n}\left(s_{n}\right)}{-R_{n}\left(s_{n}\right)+a_{1}\left(\beta_{n}\right)}\right\}
\end{array}\right.
$$

where $R_{n}(x)<0, z_{n}(x) \geq 0, \vartheta(x) \equiv \frac{\ell-s_{n}-1}{\ell-s_{n}} x-\frac{s_{n}-s_{n-1}}{\ell-s_{n}} \ell$

$$
\begin{aligned}
& R_{n}^{\prime}(x)=-R_{n}^{2}(x)-\frac{1}{\alpha^{2}}\left[\beta_{n}+2 \frac{s_{n}-s_{n}-1}{\Delta t} \frac{\ell-x}{\ell-s_{n}}\right] R_{n}(x)+\frac{1}{\alpha^{2 \Delta t}}, R_{n}(\ell)=-\beta_{n} / \alpha^{2} \\
& z_{n}^{\prime}(x)=-\left[R_{n}(x)+\frac{1}{\alpha^{2}}\left(\beta_{n}+2 \frac{s_{n}-s_{n-1}}{\Delta t} \frac{\ell-x}{\ell-s_{n}}\right)\right] z_{n}(x)-\frac{u_{n-1}(\vartheta(x))}{\alpha^{2 \Delta t}}, z_{n}(\ell)=q \beta_{n} / \alpha^{2} ; \\
& \text { (ii) } u_{c} \leq u(x, t) \leq u_{m} \\
& \text { (iii) } u_{x}(x, t) \leq 0, \text { if } u_{0}^{\prime}(x) \leq 0 .
\end{aligned}
$$

\section{NUMERICAL SIMULATION}

The following parameter is taken from a real material with a steel bar as the ablation material. The melting point is $1450^{\circ} \mathrm{C}$, and helium is the coolant.

$$
\begin{aligned}
& \ell=0.015 \mathrm{~m}, c_{\rho}=0.153 \mathrm{kcal} / \mathrm{kg} .^{\circ} \mathrm{C}, \kappa=0.0063 \mathrm{kcal} /{ }^{\circ} \mathrm{C} . \mathrm{s} . \mathrm{m} . \\
& c_{\rho L}=1.24 \mathrm{kcal} / \mathrm{kg} .{ }^{\circ} \mathrm{C}, \rho=7850 \mathrm{~kg} / \mathrm{m}^{3}, h_{r}=3500 \mathrm{kcal} / \mathrm{kg}
\end{aligned}
$$




$$
\begin{gathered}
h_{e}=1728 \mathrm{kcal} / \mathrm{kg}, q_{0}=5000 \mathrm{kcal} / \mathrm{s} . \mathrm{m}^{2}, L=65 \mathrm{kcal} / \mathrm{kg} \\
u_{m}=1450^{\circ} \mathrm{C}, u_{c}=315^{\circ} \mathrm{C}, u_{0}(x)=516^{\circ} \mathrm{C} .
\end{gathered}
$$

The numerical results are calculated through solving (35) by a FORTRAN program. The results demonstrate that if coolant is not added, the steel bar is almost melted at 10 seconds, but in the case of inputting coolant $m=2$ (the maximum coolant value in our example: $m=4.38$ ), the temperature of the outer surface never attains the melting point: the coolant is very effective.

(1) $m=0, T=10, N=30$

$\begin{array}{ccc}t_{n} & s_{N}\left(t_{n}\right) & u_{N}\left(s_{N}\left(t_{n}\right), t_{n}\right) \\ 0.3333333 & 3.823523 \mathrm{E}-003 & \\ 0.6666667 & 3.823523 \mathrm{E}-003 & 237.8863 \\ 1.0000000 & 3.823523 \mathrm{E}-003 & 1172.275 \\ 1.3333330 & 4.159586 \mathrm{E}-003 & 1450.000 \\ 1.6666670 & 4.159586 \mathrm{E}-003 & 1405.850 \\ 2.0000000 & 5.066955 \mathrm{E}-003 & 1450.000 \\ 2.3333330 & 5.066955 \mathrm{E}-003 & 993.608 \\ 2.6666670 & 5.235254 \mathrm{E}-003 & 1450.000 \\ 3.0000000 & 5.714478 \mathrm{E}-003 & 1450.000 \\ 3.3333330 & 5.714478 \mathrm{E}-003 & 1322.453 \\ 3.6666660 & 6.508625 \mathrm{E}-003 & 1450.000 \\ 4.0000000 & 6.508625 \mathrm{E}-003 & 1077.579 \\ 4.3333330 & 6.868615 \mathrm{E}-003 & 1450.000 \\ 4.6666670 & 6.868615 \mathrm{E}-003 & 1412.066 \\ 5.0000000 & 7.886279 \mathrm{E}-003 & 1450.000 \\ 5.3333330 & 7.886279 \mathrm{E}-003 & 957.884 \\ 5.6666670 & 8.059592 \mathrm{E}-003 & 1450.000 \\ 6.0000000 & 8.614007 \mathrm{E}-003 & 1450.000 \\ 6.3333340 & 8.614007 \mathrm{E}-003 & 1277.720 \\ 6.6666670 & 9.440608 \mathrm{E}-003 & 1450.000 \\ 7.0000010 & 9.440608 \mathrm{E}-003 & 1090.880 \\ 7.3333340 & 9.941348 \mathrm{E}-003 & 1450.000 \\ 7.6666680 & 9.941348 \mathrm{E}-003 & 1349.251 \\ 8.0000010 & 1.097733 \mathrm{E}-002 & 1450.000 \\ 8.3333340 & 1.097733 \mathrm{E}-002 & 985.194 \\ 8.6666670 & 1.140899 \mathrm{E}-002 & 1450.000 \\ 9.0000000 & 1.144755 \mathrm{E}-002 & 1450.000 \\ 9.3333330 & 1.276071 \mathrm{E}-002 & 1450.000 \\ 9.6666660 & 1.276071 \mathrm{E}-002 & 953.719 \\ 9.9999990 & 1.355138 \mathrm{E}-002 & \\ & & \\ & & \\ & & \\ & & \\ & & \end{array}$


(2) $m=2, T=10, N=30$

$\begin{array}{ccr}t_{n} & s_{N}\left(t_{n}\right) & u_{N}\left(s_{N}\left(t_{n}\right), t_{n}\right) \\ 0.3333333 & 2.393934 \mathrm{E}-003 & \\ 0.6666667 & 2.393934 \mathrm{E}-003 & \\ 1.0000000 & 2.393934 \mathrm{E}-003 & 661.9474 \\ 1.3333330 & 2.393934 \mathrm{E}-003 & 805.6894 \\ 1.6666670 & 2.393934 \mathrm{E}-003 & 887.0065 \\ 2.0000000 & 2.393934 \mathrm{E}-003 & 942.0304 \\ 2.3333330 & 2.393934 \mathrm{E}-003 & 983.4641 \\ 2.6666670 & 2.393934 \mathrm{E}-003 & 1016.6440 \\ 3.0000000 & 2.393934 \mathrm{E}-003 & 1044.0590 \\ 3.3333330 & 2.393934 \mathrm{E}-003 & 1068.1590 \\ 3.6666660 & 2.393934 \mathrm{E}-003 & 1087.3070 \\ 4.0000000 & 2.393934 \mathrm{E}-003 & 1104.1690 \\ 4.3333330 & 2.393934 \mathrm{E}-003 & 1117.2270 \\ 4.6666670 & 2.393934 \mathrm{E}-003 & 1137.4850 \\ 5.0000000 & 2.393934 \mathrm{E}-003 & 1142.5890 \\ 5.3333330 & 2.393934 \mathrm{E}-003 & 1146.9740 \\ 5.6666670 & 2.393934 \mathrm{E}-003 & 1151.2580 \\ 6.0000000 & 2.393934 \mathrm{E}-003 & 1155.2860 \\ 6.3333340 & 2.393934 \mathrm{E}-003 & 1158.9660 \\ 6.6666670 & 2.393934 \mathrm{E}-003 & 1162.2390 \\ 7.0000010 & 2.393933 \mathrm{E}-003 & 1167.0030 \\ 7.3333340 & 2.393933 \mathrm{E}-003 & 1174.3570 \\ 7.6666680 & 2.393933 \mathrm{E}-003 & 1173.6960 \\ 8.0000010 & 2.393933 \mathrm{E}-003 & 1173.3640 \\ 8.3333340 & 2.393933 \mathrm{E}-003 & 1173.7020 \\ 8.6666670 & 2.393933 \mathrm{E}-003 & 1174.3910 \\ 9.0000000 & 2.393933 \mathrm{E}-003 & 1175.2630 \\ 9.3333330 & 2.393934 \mathrm{E}-003 & 1176.2150 \\ 9.6666660 & 2.393934 \mathrm{E}-003 & 1177.1690 \\ 9.9999990 & 2.393933 \mathrm{E}-003 & 1178.0520\end{array}$

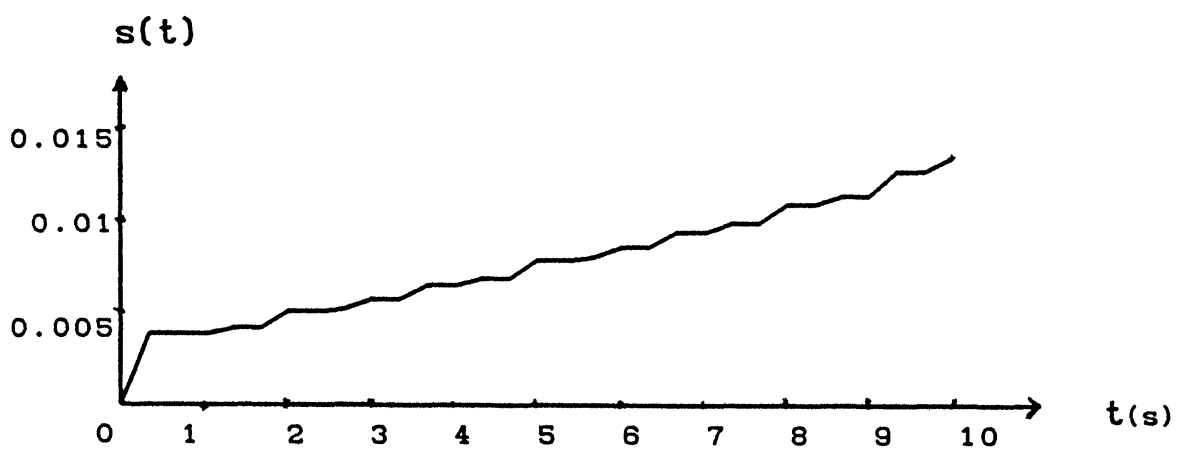

Figure 2. Numerical simulation of an ablation steel bar 


\section{REFERENCES}

[1] Yang, X.S., Transpiration cooling control of thermal protection, Acta Automatica Sinica, 11 (1985), 345-350.

[2] Yang, X.S., Transpiration cooling with control of water temperature field, Acta Automatica Sinica, 17 (1991), 385-394.

[3] Fair, H.D., Electromagnetic earth-to-space launch, IEEE Transactions on Magnetics, 25 (1989), 9-16.

[4] Palmer, M.R., Electromagnetic space launch: a re-evaluation in light of current technology and launch needs and feasibility of a near term demonstration, IEEE Transactions on Magnetics, 25 (1989), No. 1.

[5] Jiang, L.S., Remarks on the Stefan-Signorini Problem, in Free Boundary Problems: Vol. III, Res. Notes in Math. 120, 1985, 13-19.

[6] Friedman, A., and Jiang, L.S., A Stefan-Signorini Problem, J. Diff. Eqs., 51 (1984), 213-231.

[7] Friedman, A., Partial Differential Equations of Parabolic Type, Prentice-Hall, 1964.

[8] Fasano, A., Meyer, G.H., and Primicerio, M., On a problem in the Polymer Industry: Theoretical and numerical investigations of swelling, SIAM J. Math. Anal., 17 (1986), 945-960.

[9] Meyer, G.H., One-dimensional parabolic free boundary problems, SIAM, 19 (1977), 17 34.

[10] Protter, M.H. and Weinberger, H.F., Maximum Principles in Differential Equations, Springer-Verlag, 1984. 


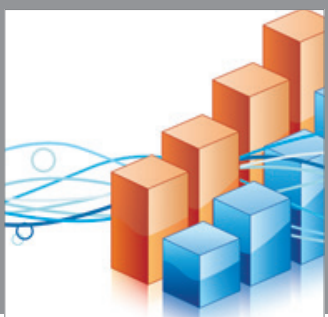

Advances in

Operations Research

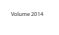

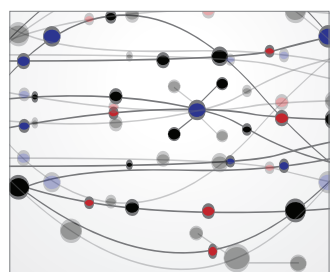

\section{The Scientific} World Journal
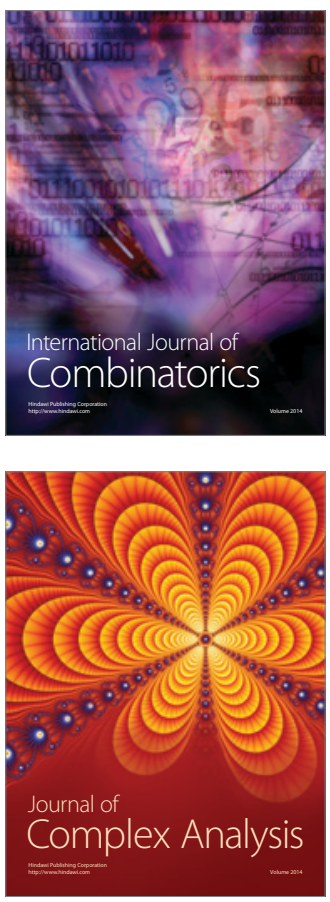

International Journal of

Mathematics and

Mathematical

Sciences
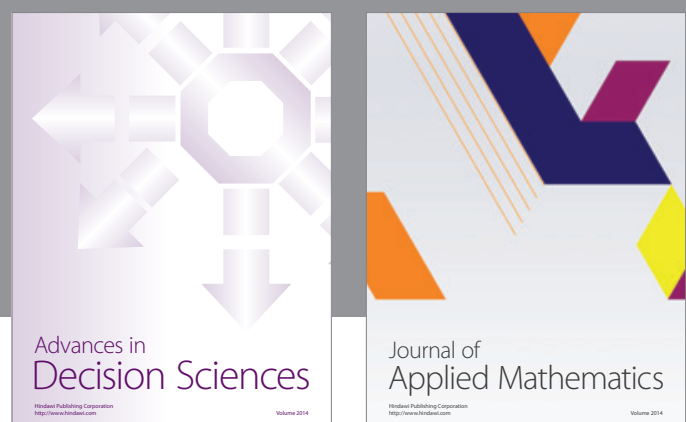

Journal of

Applied Mathematics
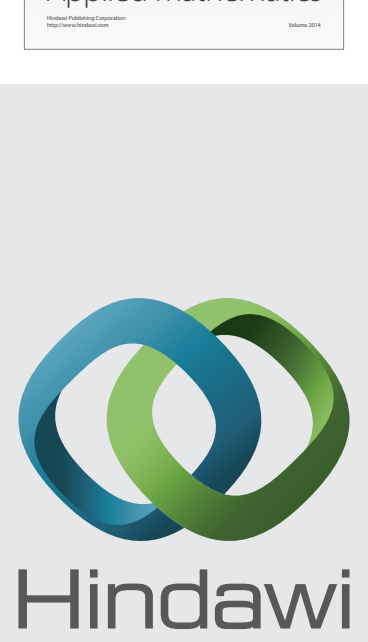

Submit your manuscripts at http://www.hindawi.com
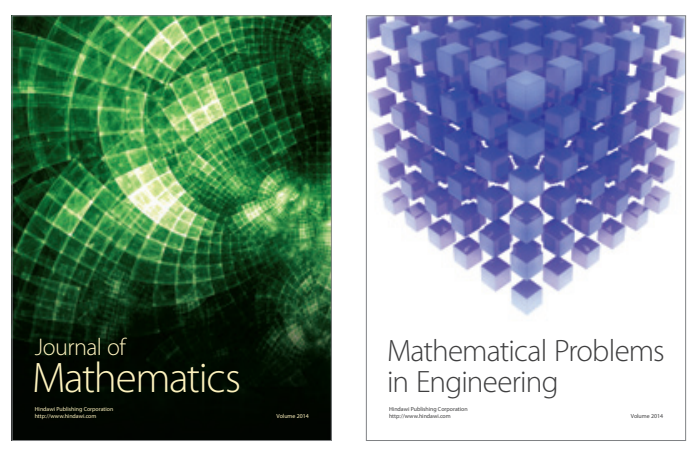

Mathematical Problems in Engineering
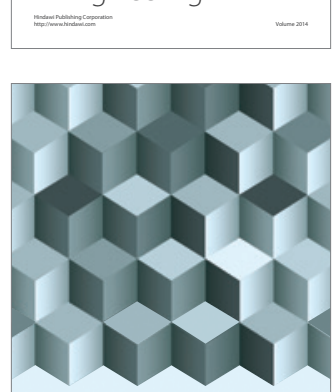

Journal of

Function Spaces
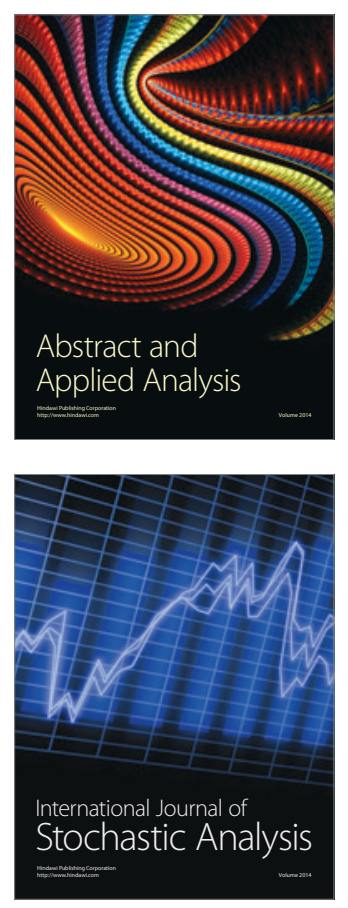

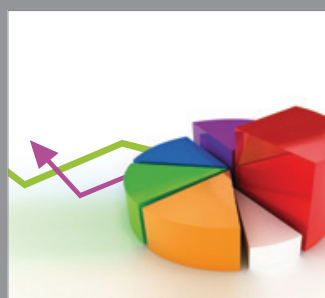

ournal of

Probability and Statistics

Promensencen
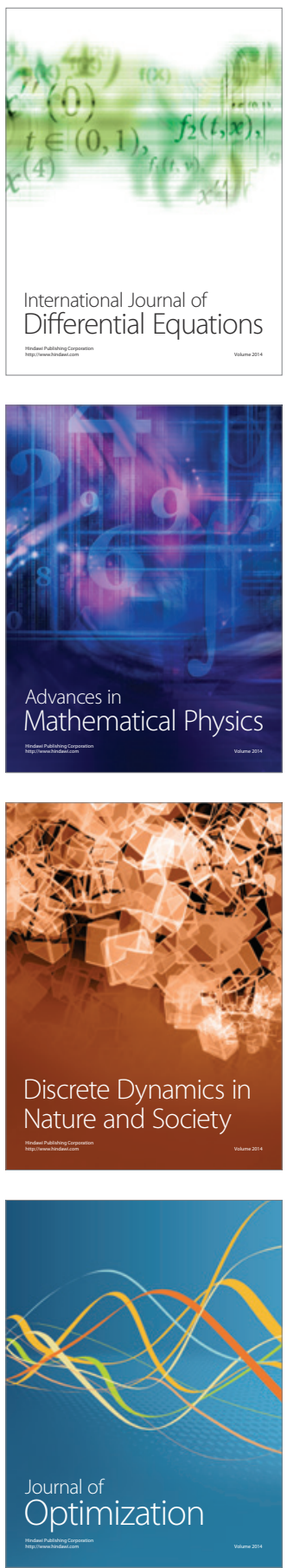